SOUZA, L.A. et al. Enterorrafias em coelhos com sutura invertida extramucosa versus continua simples contaminante e cushing: avaliação macroscópica. PUBVET, Londrina, V. 8, N. 17, Ed. 266, Art. 1773, Setembro, 2014.

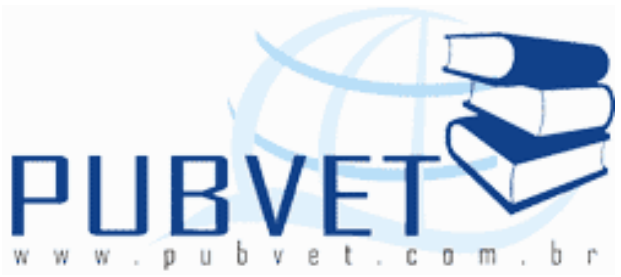

PUBVET, Publicações em Medicina Veterinária e Zootecnia.

\title{
Enterorrafias em coelhos com sutura invertida extramucosa versus continua simples contaminante e cushing: avaliação macroscópica
}

\section{Luiz Augusto de Souza ${ }^{1}$, Patrícia Maria Coletto Freitas ${ }^{2}$, Taís Andrade Dias ${ }^{3}$, Duvaldo Eurides ${ }^{4}$, Daniel Cortês Beretta ${ }^{5}$, Nadine Bou Fares ${ }^{6}$, Tamires de Paula e Silva ${ }^{6}$, Francisco Cláudio Dantas Mota ${ }^{4}$}

${ }^{1}$ Médico Veterinário. Docente da Universidade Federal de Goiás. Escola de Veterinária e Zootecnia (UFG/EVZ).E-mail: souza_vet@yahoo.com.br

${ }^{2}$ Médica Veterinária. Docente da Universidade Federal de Minas Gerais. Escola de Veterinária (UFMG/EV).

${ }^{3}$ Médica Veterinária. Doutoranda em Ciência Animal. Universidade Federal de Goiás (UFG).

4 Médico Veterinário. Docente da Universidade Federal de Uberlândia. Faculdade de Medicina Veterinária (UFU/FAMEV).

${ }^{5}$ Médico veterinário. Docente da Universidade de Rio Verde (FESURV).

${ }^{6}$ Graduanda em Medicina Veterinária. Universidade Federal de Goiás (UFG).

\section{Resumo}

O objetivo deste estudo foi avaliar e comparar macroscopicamente padrões de suturas com fio categute utilizados na rafia intestinal de coelhos. Foram utilizados 10 coelhos da raça Nova Zelândia, machos e adultos. Os animais foram submetidos a duas incisões longitudinais de $3,0 \mathrm{~cm}$ no intestino delgado, distando uma da outra a cerca de $10,0 \mathrm{~cm}$. A incisão cranial foi reparada com pontos tipo Swift extramucoso e a caudal com sutura simples contínua 
SOUZA, L.A. et al. Enterorrafias em coelhos com sutura invertida extramucosa versus continua simples contaminante e cushing: avaliação macroscópica. PUBVET, Londrina, V. 8, N. 17, Ed. 266, Art. 1773, Setembro, 2014.

contaminante e Cushing, ambas protegidas pelo omento. Decorridos dez dias de pós-operatório foi observado no local da sutura com padrão Swift menor grau de estenose e processo cicatricial mais avançado. Assim, conclui-se que a sutura extramucosa em padrão Swift pode ser indicada nas rafias intestinais de coelhos.

Palavras-chave: Coelho, Enterotorrafia, Intestino, Omento.

\title{
Enterorrhaphies in rabbits with extramucous inverted suture versus single continuous contaminant suturing and Cushing: macroscopic evaluation
}

\begin{abstract}
The aim of this study was to evaluate and compare macroscopically suture patterns with catgut thread on intestinal suture used in rabbits. Ten adult, male, New Zealand rabbits were used. In each animal two longitudinal incisions of $3,0 \mathrm{~cm}$ were made in small intestine, equidistant one from the other $10,0 \mathrm{~cm}$. The cranial incision was repaired with extramucous Swift-like suture and the caudal incision was repaired with simple continuous pattern including mucosa and the second with Cushing, both protected by omentum. After ten days postoperative was observed in Swift-like suture lower stenosis and advanced cicatricial process. It was therefore concluded that the extramucous Swift-like suture can be safety recommended in intestinal surgeries.
\end{abstract}

Keywords: Rabbit, Enterorraphy, Intestine, Omentum.

\section{INTRODUÇÃO}

Para uma sutura de boa qualidade com cicatrização rápida e sem complicação, os tecidos devem ser corretamente incisados e reconstituídos (LAZZERI, 1977). A incisão e a síntese dos tecidos devem ser orientados com sua linha de tensão a fim de facilitar a aproximação das bordas, minimizar 
SOUZA, L.A. et al. Enterorrafias em coelhos com sutura invertida extramucosa versus continua simples contaminante e cushing: avaliação macroscópica. PUBVET, Londrina, V. 8, N. 17, Ed. 266, Art. 1773, Setembro, 2014.

riscos de deiscência de pontos e não comprometer a circulação local (PAVLETIC \& PEYTON, 1991).

Diferentes modelos de suturas são utilizadas no sistema digestivo, como as contínuas e separadas em diferentes planos, usando fios sintéticos ou naturais, grampos metálicos, anéis biofragmentáveis e adesivo sintético ou biológico (FAGUNDES et al., 2001). Apesar da investigação de novos materiais para adequada coaptação das bordas de uma ferida cirúrgica e com mínima de reação inflamatória, não existe um de fio ideal (STERCHI et al., 1970). Sabe-se que os fios agem como corpos estranhos, desencadeando reação inflamatória que pode prejudicar a reparação cicatricial (FAGUNDES \& KHARMANDAYAN, 1991).

As rafias de órgão do sistema digestivo representam uma preocupação constante devido às deiscências que podem resultar em alta morbidade e mortalidade do paciente (IRVIN \& GOLINGHER, 1973). A submucosa intestinal é a camada mais importante a ser incluída na sutura por possuir maior conteúdo de colágeno (HALSTED, 1887 apud BEZUTI, 2002). As anastomoses intestinais devem ser realizadas com pontos separados, em plano único e extramucoso, para evitar penetração da agulha na luz intestinal (LAZZERI, 1997). Entretanto, suturas intestinais também são realizadas em um ou dois planos, de forma contínua ou separadas, passando por todas as camadas intestinais (FERNANDES et al., 2000). A fixação do omento maior sobre anastomoses intestinais isquêmicas promove proteção, previne deiscências e aderências, além de originar tecido de granulação e a neovascularização (ADAMS et al., 1992).

Esse trabalho teve por objetivo comparar os aspectos macroscópicos do intestino delgado de coelhos submetidos à sutura tipo Swift extramucoso e a realizada com pontos simples contínuos contaminantes e Cushing, suplementadas pela omentalização com ênfase na redução do lúmen intestinal. 
SOUZA, L.A. et al. Enterorrafias em coelhos com sutura invertida extramucosa versus continua simples contaminante e cushing: avaliação macroscópica. PUBVET, Londrina, V. 8, N. 17, Ed. 266, Art. 1773, Setembro, 2014.

\section{MATERIAL E MÉTODOS}

Foram utilizados 10 coelhos (Oryctolaus cuniculos) albinos, da raça Nova Zelândia, machos, adultos, com peso entre 1,5 e 2,0 $\mathrm{Kg}$. Os animais foram submetidos a avaliações da frequência respiratória, cardíaca, termometria e exames laboratoriais de rotina, os considerados hígidos foram utilizados no experimento.

Os animais foram pré-medicados com $0,044 \mathrm{mg} / \mathrm{Kg}$ de sulfato de atropina [Sulfato de atropina $(0,5 \mathrm{mg} / \mathrm{mL})$ - Ariston] por via subcutânea e $2,0 \mathrm{mg} / \mathrm{kg}$ de cloridrato de tramadol [Cloridrato de tramadol $(50 \mathrm{mg} / \mathrm{mL}$ ) União Química] pela via intramuscular (IM). Após 15 minutos realizou-se a indução com cetamina $\left(30,0 \mathrm{mg} / \mathrm{kg}\right.$, IM) [Dopalen ${ }^{\circledR}(100 \mathrm{mg} / \mathrm{mL})$ - VetBrands] e xilazina $(5,0 \mathrm{mg} / \mathrm{kg}, \mathrm{IM})$ [Dopaser ${ }^{\circledR}(20 \mathrm{mg} / \mathrm{mL})$ - Calier] (IM). Além disso, os animais foram submetidos à tricotomia da região abdominal e antissepsia do campo operatório com polivinil-pirrolidona [Riodine. Rioquímica Indústria Farmacêutica] e antibioticoterapia profilática com administração de cefazolina sódica $\left(30,0 \mathrm{mg} / \mathrm{kg}\right.$, IM) [Cefalotina Sódica ${ }^{\circledR}(1 \mathrm{~g})$ - Teuto] 30 minutos antes do início da intervenção cirúrgica. A manutenção anestésica foi realizada em aparelho de anestesia inalatória com uma mistura de gás oxigênio $\left[\left(\mathrm{O}_{2}\right)\right.$ Oxigênio comprimido medicinal - White Martins] e isoflurano [Forane ${ }^{\circledR}$ ABBOTT] por meio de vaporizador universal com máscara em sistema aberto tipo Baraka [Multiagente Modular 1410 - TAKAOKA].

Foi praticada uma incisão mediana retro-umbilical de aproximadamente de $10 \mathrm{~cm}$ para exposição proximal do duodeno, onde foram realizadas duas incisões de aproximadamente $3,0 \mathrm{~cm}$ até o lúmen intestinal, distando $10 \mathrm{~cm}$ uma da outra. A cranial foi aproximada com pontos tipo Swift extramucoso e a incisão caudal reconstituída com sutura simples contínua contaminante seguida do ponto de Cushing. As suturas foram realizadas com categute cromado no 4-0 [Categute cromado - Laboratório Bruneau] e o omento maior fixado sobre as suturas. A parede abdominal foi reaproximada com pontos em "X" com fio categute cromado no 3-0 e a pele com fio náilon no 3-0 [Náilon monofilamento preto - Brasmédica] por pontos simples separados. 
SOUZA, L.A. et al. Enterorrafias em coelhos com sutura invertida extramucosa versus continua simples contaminante e cushing: avaliação macroscópica. PUBVET, Londrina, V. 8, N. 17, Ed. 266, Art. 1773, Setembro, 2014.

No pós-operatório (PO) os coelhos foram mantidos em gaiolas individuais. Decorridos 6 horas do PO, os animais receberam ração liquefeita até o $3^{\circ} \mathrm{dia}$. A partir do $3^{\circ}$ até o $6^{\circ} \mathrm{dia}$ foi fornecida ração pastosa umedecida em água e gradativamente a ração sólida introduzida até o $10^{\circ}$ dia de PO. Diariamente foram realizados curativos das feridas de pele com solução salina $0,9 \%$ e polivil-pirrolidona $0,1 \%$ por sete dias, antibióticoterapia com cefazolina sódica $(30 \mathrm{mg} / \mathrm{kg}, \mathrm{IM})$ de 12 em 12 horas, durante cinco dias e flunixina-meglumina $\left[\right.$ Meflosyl ${ }^{\circledR}(50 \mathrm{mg} / \mathrm{mL})$ - Fort Dodge] na dose de $1,0 \mathrm{mg} / \mathrm{Kg}$, uma vez ao dia durante dois dias.

No $10^{\circ}$ dia de PO os animais foram submetidos à eutanásia com sobredose de tiopental sódico [Tiopental. Cristália-Produtos Químicos Farmacêuticos] e cloreto de potássio a 19,1\% [Cloreto de potássio 19,1\% Samtec Biotecnologia] conforme recomenda o código de ética para uso de animais em pesquisa científica (CRMV, 2002). Durante a avaliação macroscópica optou-se por identificar principalmente a presença de fístulas, abscessos, aderências, deiscências e estenoses do lúmen intestinal.

\section{RESULTADOS E DISCUSSÃO}

A boa evolução clínica dos animais e a ausência de anormalidades à necropsia confirmaram a adequação da anastomose em plano único quando comparada com a técnica em dois planos.

As técnicas cirúrgicas que envolvem o trato digestivo são classificadas como procedimentos limpos contaminados ou contaminados, devido ao alto número de bactérias patológicas que habitam trato gastrointestinal (SANTOS et al., 2007). Este fator interfere ativamente no balanço entre a síntese e deposição de colágeno e, consequentemente, na cicatrização. Portanto, o antibiótico cefazolina de forma profilática, conforme sugerido por FOSSUM (2002) evitou alterações que pudessem estar relacionadas à infecção local.

A realimentação líquida por via oral, nas primeiras horas de pósoperatório, como utilizado por AGUILAR-NASCIMENTO e GÖELZER (2002) no homem submetido à enterorrafia não interferiu no processo cicatricial das 
SOUZA, L.A. et al. Enterorrafias em coelhos com sutura invertida extramucosa versus continua simples contaminante e cushing: avaliação macroscópica. PUBVET, Londrina, V. 8, N. 17, Ed. 266, Art. 1773, Setembro, 2014.

incisões intestinais. Esta conduta de alimentação foi segura, por não ter sido observado deiscência nos dois métodos empregados.

A escolha do período em que os animais foram submetidos à eutanásia referente ao décimo dia de PO. Neste período, os coelhos já se alimentavam com dieta sólida que proporcionava maior tensão nas linhas de suturas. Foi referido por REZENDE NETO \& SILVA (1999) que os pontos de suturas utilizados somado a omentalização são suficientes para resistir à tensão ocasionada pela presença do alimento, o que corrobora com os achados deste estudo. A presença do próprio conteúdo intraluminar, além do contato direto com a sutura, promove forças de cisalhamento que interferem no processo. A distensão local pelo conteúdo também pode desencadear isquemia na área suturada conforme afirmou SANTOS et al. (2007).

O período de observação foi suficiente para verificar possíveis complicações decorrentes dos métodos utilizados. Não sendo constadas alterações locais como peritonite, abscessos e fístulas que podem ser notadas nas cirurgias do trato gastrointestinal, como relatou AGUILAR-NASCIMENTO \& GÖELZER, (2002).

Durante a necrópsia não foram evidenciadas anormalidades. As aderências peritoneais se equivaleram em ambas as faces da anastomose e nas linhas de suturas notava-se hipermia da mucosa, porém com boa reparação da serosa. Também foram observadas aderências entre o local da omentalização com outras partes do intestino delgado, entretanto, o peristaltismo manteve-se normal. Sabe-se que a manipulação do omento maior pode ocasionar isquemia e hemorragia tendo como consequência as aderências intestinais (Figura 1A) afirmou GRECA et al. (1998) e constatado no presente estudo. Embora o fio categute cromado possa ser tolerado pelos tecidos (KNECHT et al., 1985; FATURETO \& TEIXEIRA, 1993), em todos os animais foram observadas hiperemia, edema e intensa vascularização ao redor das linhas de suturas, tanto com o tipo Swift extramucoso como a simples contínuo associado ao Cushing (Figura 1B). 
SOUZA, L.A. et al. Enterorrafias em coelhos com sutura invertida extramucosa versus continua simples contaminante e cushing: avaliação macroscópica. PUBVET, Londrina, V. 8, N. 17, Ed. 266, Art. 1773, Setembro, 2014.
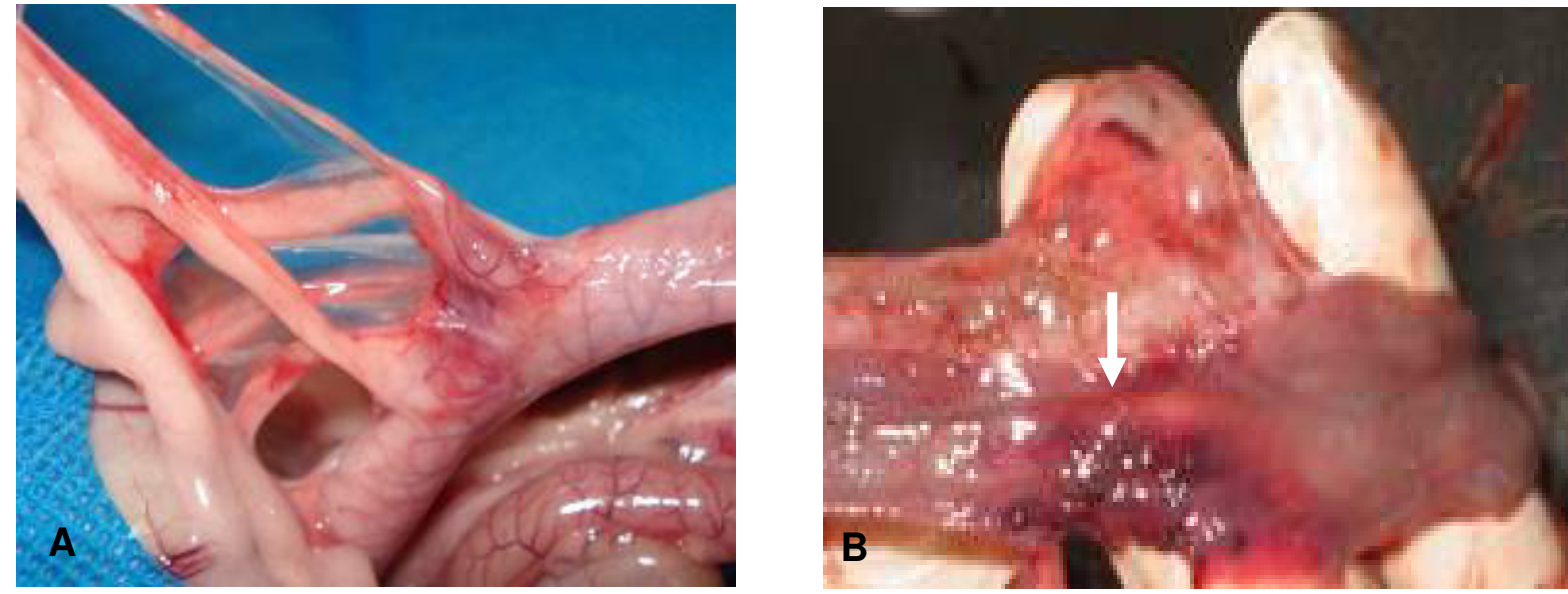

Figura 1: Aspectos macroscópicos da enterorrafia em coelhos com sutura de Swift decorridos 10 dias de pós-operatório. Notar em (A) processo de ometalização caracterizado por aderência no local da enterorrafia. Em (B) observar a presença de hiperemia, intensa vascularização e edema caracterizando processo inflamatório (seta).

A sutura em um plano com pontos isolados Swift, não interferiu de forma significativa no diâmetro do lúmen intestinal. No entanto, nos animais submetidos a dois planos de sutura (figuras 2 e 3 ) a estenose foi parcial devido à sutura invaginante não contaminante que ocasiona diminuição do diâmetro do lúmen intestinal (FOSSUM, 2002). Fato este não observado no local da sutura extramucoso com Swift. Entretanto, durante o período de observação não foram verificadas manifestações clínicas que pudessem estar relacionadas com as estenoses.

Com os dois tipos de síntese a mucosa intestinal se apresentava macroscopicamente reconstituída. Entretanto, no local da aproximação com a sutura de Swift, foi notado que as bordas da incisão se eram mais regulares. Possivelmente, a sutura extramucosa não ocasionou maiores traumatismos na mucosa e não interferir no processo de cicatrização intestinal (SHIMIZU et al., 2000). 
SOUZA, L.A. et al. Enterorrafias em coelhos com sutura invertida extramucosa versus continua simples contaminante e cushing: avaliação macroscópica. PUBVET, Londrina, V. 8, N. 17, Ed. 266, Art. 1773, Setembro, 2014.
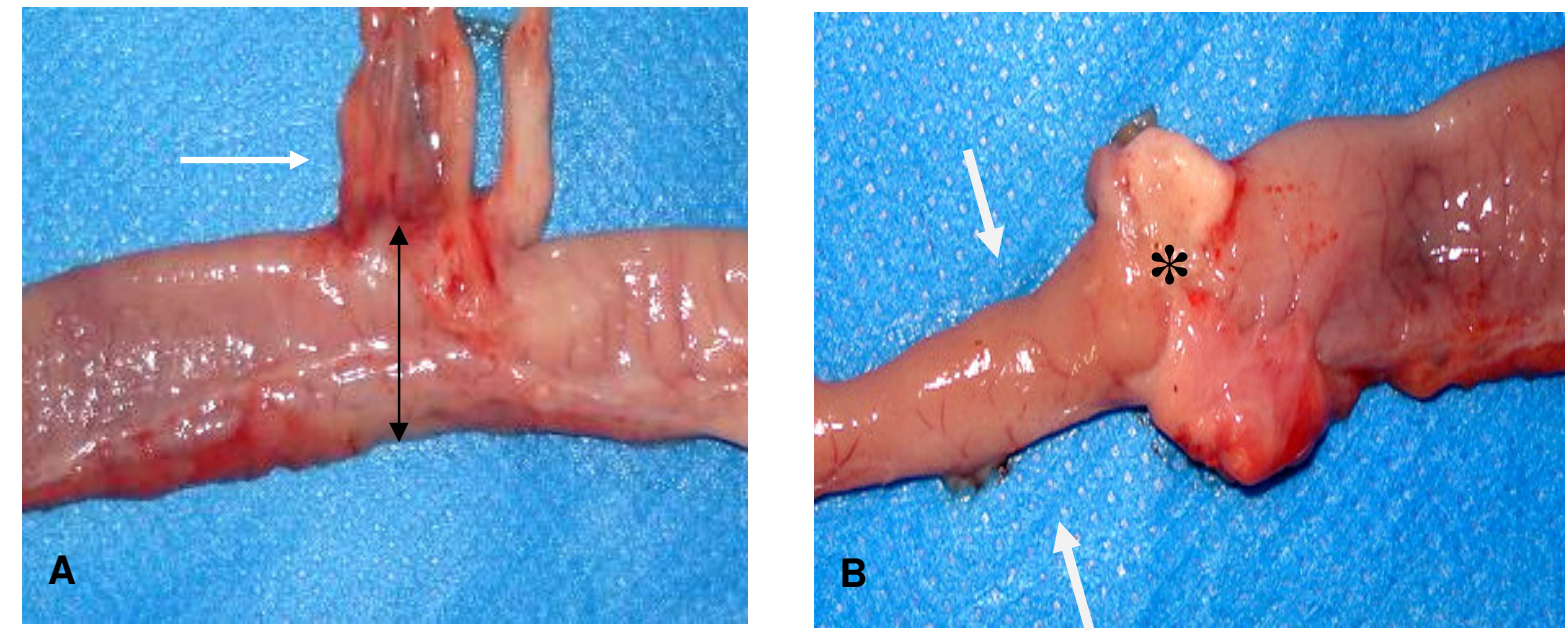

Figura 2: Aspectos macroscópicos da enterorrafia em coelhos decorridos 10 dias de pós-operatório. Notar em (A) sutura com pontos Swift, seta branca aponta o local da omentalização e seta preta ausência de estenose intestinal. Em (B) padrão de sutura em duas linhas, simples contínua e Cushing. Nota-se intensa estenose intestinal (setas brancas), após local da enterorrafia (asterisco).

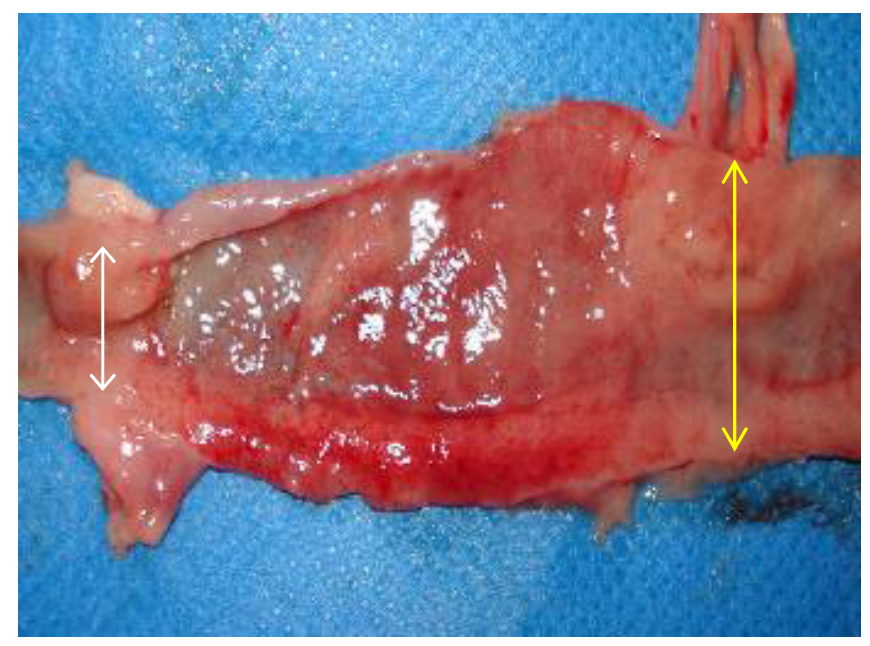

Figura 3: Aspectos macroscópicos da enterotomia em coelhos decorridos 10 dias de pós-operatório. Observar o grau de estenose provocado pela utilização da sutura simples continua associada ao padrão Cushing, seta branca e seta amarela para os pontos Swift. 
SOUZA, L.A. et al. Enterorrafias em coelhos com sutura invertida extramucosa versus continua simples contaminante e cushing: avaliação macroscópica. PUBVET, Londrina, V. 8, N. 17, Ed. 266, Art. 1773, Setembro, 2014.

Nas condições em que este estudo foi desenvolvido conclui-se que a sutura extramucosa do tipo Swift pode ser aplicada nas rafias intestinais de coelhos, pois proporcionou menor grau de estenose e moderado processo inflamatório, quando comparada à sutura em dois planos realizada com pontos simples contínuos e Cushing que é utilizada em ráfias intestinais.

\section{REFERÊNCIAS BIBLIOGRÁFICAS}

ADAMS, W.; CTERCTEKO, G.; BILOUS, M. Effect of an omental wrap on the healing and vascularity of compromised intestinal anastomoses. Diseases of the colon and rectum, Philadelphia, v. 35, p. 731-738, 1992.

AGUILAR-NASCIMENTO, J. E.; GÖELZER, J. Alimentação precoce após anastomoses intestinais: riscos ou benefícios? Revista da Associação Médica Brasileira, São Paulo, v. 48, n. 4, 2002.

BEZUTI, M. T.; FERES, O.; CAMPOS, A. D.; APRILLI, F.; ROCHA, J. J. R.; GARCIA, R. S.; JOVILIANO, O. F. D. Cicatrização de anastomoses colonicas na vigência de obstrução intestinal. Estudo experimental em ratos. Acta Cirúrgica Brasileira, São Paulo, v.17, supl.3, 2002.

CRMV. Conselho Regional de Medicina Veterinária. EUTANÁSIA: Resolução do CFMV institui normas e procedimentos para eutanásia em animais. Revista Veterinária e Zootecnia em Minas Gerais, Belo Horizonte, v. 75, n. 17, p. 25, 2002.

FAGUNDES, D. J.; TAHA, M.; MONTEIRO, S. E. F. Adesivos em cirurgia. In: Condutas em Cirurgia, São Paulo: Editora Atheneu, 2001. p. 669-674.

FAgundes, D. J. E.; KHARMAndAyAn, P. O Fio Cirúrgico. Acta Cirúrgica Brasileira, São Paulo, v. 6, n. 4, p. 177-181, 1991.

FATURETO, M. C.; TEIXEIRA, V. P. A. Reação inflamatória a fios de sutura. In: HERING, F. L. O.; FABOR, S.; ROSEMBERG, D. (Eds) Bases técnicas e teóricas de fios de suturas, São Paulo: Roca, 1993, p. 31-39.

FERNANDES, L. C.; MATOS, M. D.; NOVELLI, S. B. Estudo comparativo entre anastomoses intestinais com sutura manual e com anel biofragmentável em cães sob a administração de corticosteróides. Revista da Associação Médica Brasileira, São Paulo, v. 46, n. 2, p. 113$120,2000$.

FOSSUM, T. W. Cirurgia do intestino delgado. Cirurgia de pequenos animais, São Paulo: Roca, 2002. 1408 p.

GRECA, F. H.; BIONDO-SIMÕES, M. L. P.; SOUZA-FILHO, Z. A.; SILVA A. P. G.; NASSIF, A. E.; COSTA P. B. Effects of omental wrapping on the colonic anastomoses: experimental study in rats. Acta Cirúrgica Brasileira, São Paulo, v. 13, n. 3, 1998.

IRVIN, T. T.; GOLINGHER, J. C. A etiology of disruption of intestinal anastomosis. British Journal of Surgery, Guildford, v. 60, p. 461-464, 1973. 
KNECHT, C. D.; ALLEN, A. R.; WILLIANS, D. J. Técnicas fundamentais em cirurgia veterinária, São Paulo: Roca, 1985. 308 p.

LAZZERI, L. Fases fundamentais da técnica cirúrgica, São Paulo: Varela Livros, 1977. 190 p.

PAVLETIC, M. M.; PEYTON, L. C. Cirurgia plástica e reconstrutiva nos cães e gatos. In: Bojrab, M. J. (Eds). Cirurgia dos pequenos animais, 2ed. São Paulo: Roca, 1991. p. 461-471.

REZENDE NETO, J. B.; SILVA, A. L. Avaliação da resistência de suturas duodenais com ou sem omentoplastia pediculada: estudo experimental em ratas. Acta Cirúrgica Brasileira, São Paulo, v. 14, n. 3, 1999.

SANTOS, C. L.; ABÍlio, E. J.; OLIVEIRA, A. L. A.; MARIANO, C. M. A.; NUNES, A. T.; CARVALHO, E. C. Q. Estudo comparativo entre as técnicas de sutura total e serosubmucosa em anastomoses colônicas na presença de um protetor intraluminar em cães. Revista do Colégio Brasileiro de Cirurgiões, Rio de Janeiro, v. 34, n. 4, p. 245-250, 2007.

SHIMIZU, J. B.; FARIA, M. A. R.; BELETTI, M. E. PEREIRA, K. C.; MOTA, F. C. D.; MASTRANTONIO, E. C.; FREITAS, P. M. C.; ALVES, K. A. Emprego de fio naylon monofilamentoso em sutura simples contínua e sutura com cola tecidual histoacryl, em intestino delgado de cão- estudo comparativo. Ciência Animal Brasileira, Goiânia, v. 1, p. $34,2000$.

STERCHI, M.; JAMES, P. M.; MYERS, R. T. Cyanoacrylate monomers in anastomosis of irradiated intestine preventing anastomotic leaks. Surgical forum, Chicago, v. 21, p. 332334, 1970. 